\title{
A five year follow-up of lung function among chemical workers using flow-volume and impedance measurements
}

\author{
S. Keman*, B. Willemse*, G.J. Wesseling**, E. Kusters ${ }^{+}$, P.J.A. Borm*
}

A five year follow-up of lung function among chemical workers using flow-volume and impedance measurements. S. Keman, B. Willemse, G.J. Wesseling, E. Kusters, P.J.A. Borm. C ERS Journals Ltd 1996.

ABSTRACT: Impedance of the respiratory system, measured using the forced oscillation technique (FOT), has repeatedly been proposed as a tool for occupational health screening.

The aim of this study was to compare the outcome of impedance measurements and flow-volume curves, and to study relationships between lung function decline and specific exposures and smoking. Both measurements were applied in 136 chemical workers from seven main production/personnel groups during a 5 year followup (1990-1995).

The prevalence of airway obstruction (forced expiratory volume in one second (FEV1) $<80 \%$ of predicted) at both time-points was about $5 \%$ but did not necessarily include the same subjects. The average decline in FEV1 over 5 yrs was 200 $\mathrm{mL}$ (predicted decline $160 \mathrm{~mL}$ ). Although most impedance parameters correlated to flow-volume parameters in both years, a rapid 5 year decline of FEV1 ( $>330 \mathrm{~mL}$ ) was only accompanied by a significant decrease in the reactance at $8 \mathrm{~Hz}(\mathrm{X8})$ and an increase in the resonance frequency $(f 0)$. Interestingly, decline in FEV1 was significantly dependent on age, years of employment, pack-years, FEV1, and all impedance parameters at the start in $1990(\mathrm{r}=0.35 ; \mathrm{p}=\mathbf{0 . 0 1})$. Similarly, the increase in $f_{0}$ was significantly dependent on age, years of employment, pack-years, $f 0, F E V 1$, forced vital capacity (FVC), peak expiratory flow (PEF) and maximal mid-expiratory flow $(M M E F)$ at the start $(r=0.56 ; p<0.01)$. Those with a rapid decline in FEV1 were relatively more represented among acid workers (relative risk $(R R)=2.14)$, maintenance workers $(R R=2.1)$ and bulk/transport workers $(R R=1.78)$, but none of these was significant. Lung function, however, was significantly lower in the bulk/transport workers $(n=14)$ compared to the rest, based both on flow-volume (FEV1, FVC) and impedance $\left(X 8, f_{0}\right)$ measurements. Smoking had a significant effect on most flow-volume parameters but only for frequency dependence among the impedance outcomes.

It is concluded that alterations in impedance parameters do reflect changes in flow-volume curves induced by age, smoking and occupational exposure. Therefore, these data are a valuable extension to current cross-sectional data.

Eur Respir J., 1996, 9, 2109-2115.
Depts of *Health Risk Analysis and Toxicology, and **Pulmonology, University of Limburg, Maastricht, The Netherlands. +Free University of Brussels, Brussels, Belgium.

Correspondence: P.J.A Borm

Dept of Health Risk Analysis and Toxicology University of Limburg

P.O. Box 616

6200 MD Maastricht

The Netherlands

Keywords: Forced oscillation technique irritants

lung function decline

spirometry

Received: December 121995

Accepted after revision May 231996
Although a decline in forced expiratory volume in one second (FEV1) is generally considered as a gold standard to detect airflow limitation, different (mechanical) alterations of the respiratory system can influence this parameter. Impedance measurements of the respiratory system using the forced oscillation technique (FOT) can provide additional information on the mechanical characteristics of the respiratory system [1,2]. Firstly, it was demonstrated that impedance measurements are sensitive in detecting airflow limitation induced by specific or nonspecific challenge tests $[3,4]$ and in patients with asthma [5] and chronic obstructive pulmonary disease (COPD) [6]. Secondly, in the past decade, several crosssectional investigations $[2,7,8]$ have shown that impedance measurements also offer a simple noninvasive method to detect airflow limitation in different occupational settings. Some of these studies showed that the
FOT provided better sensitivity to distinguish between subjects with respect to exposure to irritant gases [7], organic dust and endotoxin [9], or to detect mild obstructive patterns in underground coal workers without apparent radiographic abnormalities [8]. However, the pattern of impedance measurements and in particular its comparison with flow-volume parameters is highly dependent both on type and extent of exposure [2]. Although we and others have proposed the FOT as a tool for epidemiology of occupational lung diseases $[2,8,10]$, few longitudinal data on impedance measurements are available. Therefore, we performed a follow-up study of lung function among workers of a large chemical plant, mainly exposed to low levels of respiratory irritants, using flowvolume and impedance measurements.

The objectives of this study were: 1) to relate alterations in impedance parameters measured using the FOT 
to changes in flow-volume parameters in workers exposed to chemicals; and 2) to study whether lung function (decline) was related to specific exposure categories.

\section{Methods}

\section{Study design and protocol}

This study was designed as a 5 year (1990-1995) follow-up study. Male workers from a large chemical plant, who came to the occupational physician for a routine annual medical check-up in April and May 1990, were included in the study after providing written informed consent $(n=160)$. All subjects had to complete three impedance measurements and three flow-volume curves. Impedance measurements were always performed before flow-volume curves to avoid the influence of forced inspiratory manoeuvres on the bronchial tonus [11]. At the beginning of 1995, all subjects were asked to participate in this study, and then in the months of April and May 1995 the same procedure was performed after evaluation of a self-administered Dutch version of the British Medical Research Council (BMRC) standardized questionnaire on respiratory symptoms, smoking habits, and additional questions concerning working history in the second phase of study. Twenty four of the 160 workers did not participate in this study for various reasons (three no longer employed, 17 not satisfied with the first study in 1990, and four ill at the time of study). Finally, 136 complete data-sets were obtained and analysed. Monitoring data were available for each production process but these were not used because of the complexity of multiple exposure and the limited number of subjects. Seven main production processes/personnel groups were distinguished: chemicals, acids, polymers, polyurethanes, bulk/transport and maintenance personnel, and others. Bulk/transport workers are mainly exposed to nitrate, phosphate and potassium (NPK)-containing dust during loading and scraping.

\section{Lung function measurements}

Forced expiratory flow-volume curves were recorded according to European Coal and Steel Community (ECSC) [12] criteria using a Vicatest P1 dry spirometer (Mijnhardt, Bunnik, The Netherlands). Three recordings within 5\% of forced vital capacity (FVC) or a $100 \mathrm{~mL}$ range were obtained from each subject. Parameters derived from the flow-volume curves were: FVC, FEV1, FEV1/FVC ratio, peak expiratory flow (PEF), maximal mid-expiratory flow (MMEF), and maximal expiratory flow after 50 and $75 \%$ exhalation (MEF50 and MEF25). The first four parameters are the effort-dependent part and the last three are the effort-independent part of the flowvolume curve. The FVC, FEV1 and FEV1/FVC ratio are used to describe the airway obstruction in the large airways, whereas MMEF values are used to describe airway obstruction in the small airways. All flow-volume values were related to the reference values of the European Coal and Steel Community (ECSC) for individual diagnosis [12]. A deflection value of two or more standard deviations (i.e. a value less than $80 \%$ of the standard value) of FEV1 reflected airway obstruction. The spirometer was calibrated twice daily and all subjects were measured on the same spirometer by the same investigator on both occasions.

Impedance of the respiratory system was measured using the FOT originally described by LÀNDSÉR et al. [1]. The FOT measures impedance parameters of the respiratory system, namely resistance $(R)$ and reactance $(X)$. The seated subject, wearing a noseclip, supported the cheeks with his hands and breathed quietly via a tube. A pseudorandom noise pressure signal containing all harmonics of $2 \mathrm{~Hz}$ from 4 to $48 \mathrm{~Hz}$ was applied at the mouth by means of a loudspeaker. Recorded pressure and flow signals were analysed by spectral analysis techniques. Mean values of three successive measurements, each lasting $8 \mathrm{~s}$ were used in the analysis. Of the impedance data obtained, the resistance at $8 \mathrm{~Hz}(R 8)$, at $28 \mathrm{~Hz}(R 28)$, the difference between $R 28$ and $R 8$ (frequency dependence (FD) of resistance, signifying the course of the resistance versus frequency curve), the reactance at $8 \mathrm{~Hz}\left(X_{8}\right)$ and the resonant frequency ( $f 0$, the frequency at which $X$ equals zero) were used for analysis. $R 8$ was chosen because usually at this frequency coherence function $>0.95$ was obtained, which was not always obtained at lower frequencies. A negative FD and a resonance frequency $(f 0)$ exceeding $15 \mathrm{~Hz}$ were regarded as indicative of airway obstruction. The equipment was calibrated daily and all measurements were performed using the same apparatus during both study episodes.

Short-term variability of impedance of the respiratory system was tested among 10 healthy subjects for 3 weeks, 3 days $\cdot$ week $^{-1}$, and showed variation coefficients of $10,15,137$, and $14 \%$ for $R 8, X 8, \mathrm{FD}$, and $f 0$, respectively. Long-term variability was derived from a followup study among 10 grain workers [13], showing similar values for the above parameters at 2 yrs follow-up.

\section{Statistical analysis}

Pack-years were calculated from the questionnaires, as average daily of total number of packs of cigarettes smoked multiplied by the number of years smoked. Average lung function data for the same groups in 1990 and 1995 were tested for significance using the Student's t-test for paired samples. Correlations of flow-volume curves with impedance parameters were calculated by Pearson's correlation test. Relationships between changes in FEV1 in the quartile's groups and alterations in impedance parameters were tested by one-way analysis of variance (ANOVA) within all groups and between individual groups and the others. Characteristics and lung function outcomes of workers from different plants in 1995 and 1990 were tested by one-way ANOVA. To determine the strength of the association between presence of a rapid decline in FEV1 and the main production process, relative risks (RRs) were defined as subjects included in the upper quartile (group IV) or the group of rapid decline in FEV1 and controls as the remainder of the population studied. The influences of smoking habits on lung function parameters were tested by one-way ANOVA. Probability values less than 0.05 were considered as statistically significant. All procedures were performed using Statistical Package for the Social Sciences (SPSS) for Windows 6.0 software. 


\section{Results}

\section{General data}

At follow-up, most flow-volume parameters showed a significant decrease compared to the starting point ( $\mathrm{t}$-test for paired samples, $\mathrm{p}=0.01$ ). A strong correlation between both time-points was observed for most flow-volume parameters (table 1). Average values of flow-volume parameters were not different from normal (reference) values in both years, and average decline over 5 yrs was: FVC $90 \mathrm{~mL}$; FEV1 $200 \mathrm{~mL}$; FEV1/FVC ratio 2.5\%; and MMEF $0.56 \mathrm{~L} \cdot \mathrm{s}^{-1}$. The average decline in FEV1 of 40 $\mathrm{mL} \cdot \mathrm{yr}^{-1}$ did not differ much from the predicted decline of $32 \mathrm{~mL} \cdot \mathrm{yr}^{-1}$ from the ECSC equations [12]. The estimated decline was predicted on a 5 year increase of age with height and weight remaining the same. Impedance parameters showed similar outcomes to the flow-volume curves. Average values of impedance parameters could also be considered as normal values in both years. Most flow-volume parameters were significantly decreased between 1990 and 1995, whilst only FD reached significance among impedance values. Although not significant, the increase in $R 8\left(0.37 \mathrm{hPa} \cdot \mathrm{s} \cdot \mathrm{L}^{-1}\right)$ and $f_{0}(0.32 \mathrm{~Hz})$ are in line with the above data. Correlation coefficients

Table 1. - Flow-volume and impedance parameters in the same group of chemical workers $(n=136)$ in 1990 and 1995

\begin{tabular}{|c|c|c|c|}
\hline Parameter & 1990 & 1995 & $\begin{array}{l}\text { Correlation } \\
\text { coefficient }\end{array}$ \\
\hline Age yrs & 36 & 41 & \\
\hline Height $\mathrm{cm}$ & $177 \pm 7$ & $177 \pm 7$ & \\
\hline Weight $\mathrm{kg}$ & $78 \pm 10$ & $80 \pm 10$ & \\
\hline \multicolumn{4}{|l|}{ Smokers $\mathrm{n}$} \\
\hline Current & 39 & 36 & \\
\hline Past & 48 & 51 & \\
\hline Lifetime NS & 49 & 49 & \\
\hline \multicolumn{4}{|l|}{ Smoking habit } \\
\hline Current pack-yrs & ND & 16.8 & \\
\hline Past pack-yrs & ND & 9.2 & \\
\hline \multicolumn{4}{|l|}{ Flow volume } \\
\hline FVC L & $5.25 \pm 0.70$ & $5.16 \pm 0.73 * *$ & 0.91 \\
\hline FVC $\%$ pred & $107 \pm 10$ & $110 \pm 31$ & 0.30 \\
\hline FEV1 L & $4.02 \pm 0.60$ & $3.82 \pm 0.62 * *$ & 0.94 \\
\hline FEV1 $\%$ pred & $98 \pm 11$ & $97 \pm 11 * *$ & 0.86 \\
\hline FEV1/FVC \% & $77 \pm 6$ & $74 \pm 6 * *$ & 0.76 \\
\hline PEF $\quad L \cdot \mathrm{s}^{-1}$ & $8.85 \pm 1.64$ & $8.65 \pm 1.46$ & 0.61 \\
\hline PEF \% pred & $93 \pm 16$ & $93 \pm 15$ & 0.56 \\
\hline MMEF $\mathrm{L} \cdot \mathrm{s}^{-1}$ & $3.73 \pm 1.03$ & $3.17 \pm 0.97 * *$ & 0.84 \\
\hline MMEF \% pred & $81 \pm 21$ & $72 \pm 20 * *$ & 0.80 \\
\hline \multicolumn{4}{|l|}{ Impedance } \\
\hline$R 8 \quad \mathrm{hPa} \cdot \mathrm{s} \cdot \mathrm{L}^{-1}$ & $2.30 \pm 0.74$ & $2.67 \pm 2.51$ & 0.23 \\
\hline$X 8 \mathrm{hPa} \cdot \mathrm{s} \cdot \mathrm{L}^{-1}$ & $0.15 \pm 0.25$ & $0.16 \pm 0.30$ & 0.53 \\
\hline $\mathrm{FD} h \mathrm{hPa} \cdot \mathrm{s} \cdot \mathrm{L}^{-1}$ & $0.53 \pm 0.48$ & $0.27 \pm 0.63 * *$ & 0.32 \\
\hline$f_{0} \mathrm{~Hz}$ & $7.64 \pm 2.81$ & $7.96 \pm 2.99$ & 0.45 \\
\hline
\end{tabular}

Values are presented as mean \pm SD. FVC: forced vital capacity; $\%$ pred: percentage of predicted value; FEV1: forced expiratory volume in one second; PEF: peak expiratory flow; MMEF: maximal mid-expiratory flow; NS: nonsmoker; $R 8$ : resistance at $8 \mathrm{~Hz} ; X 8$ : reactance at $8 \mathrm{~Hz}$; FD: frequency dependence $(R 28-$ $R 8) ; f 0:$ resonance frequency (frequency where reactance is equal to zero); ND: not determined. \#: correlation coefficients between individual values for 1990 and 1995. Significances were tested by t-test for paired samples. $* *: \mathrm{p}<0.01,1990 \mathrm{vs}$ 1995.
Table 2. - Correlation coefficients of flow-volume and impedance parameter measurements in 136 chemical workers in 1990 and 1995

\begin{tabular}{|c|c|c|c|c|c|}
\hline \multicolumn{2}{|l|}{ Parameter } & $\begin{array}{c}R 8 \\
\mathrm{hPa} \cdot \mathrm{s} \cdot \mathrm{L}^{-1}\end{array}$ & $\begin{array}{c}X 8 \\
\mathrm{hPa} \cdot \mathrm{s} \cdot \mathrm{L}^{-1}\end{array}$ & $\underset{\mathrm{hPa} \cdot \mathrm{s} \cdot \mathrm{L}^{-1}}{\mathrm{~F}}$ & $\begin{array}{l}f_{0} \\
\mathrm{~Hz}\end{array}$ \\
\hline \multirow[t]{2}{*}{ FVC L } & 1990 & $-0.22 * *$ & $0.35 * *$ & $0.27 * *$ & $-0.26 * *$ \\
\hline & 1995 & $-0.22 * *$ & $0.29 * *$ & $0.26 * *$ & $-0.22 * *$ \\
\hline \multirow[t]{2}{*}{ FEV1 L } & 1990 & $-0.30 * *$ & $0.36 * *$ & $0.33 * *$ & $-0.26^{* *}$ \\
\hline & 1995 & $-0.27 * *$ & $0.32 * *$ & $0.32 * *$ & $-0.23 * *$ \\
\hline \multirow[t]{2}{*}{ FEV1/FVC \% } & 1990 & $-0.17 *$ & 0.07 & 0.14 & -0.06 \\
\hline & 1995 & -0.12 & 0.12 & 0.16 & -0.06 \\
\hline \multirow[t]{2}{*}{ PEF $\quad L \cdot s^{-1}$} & 1990 & -0.15 & 0.09 & 0.11 & -0.07 \\
\hline & 1995 & -0.12 & 0.15 & 0.04 & -0.14 \\
\hline \multirow[t]{3}{*}{ MMEF $\quad L \cdot s^{-1}$} & 1990 & $-0.29 * *$ & $0.24 * *$ & $0.24 * *$ & -0.15 \\
\hline & 1995 & $-0.22 * *$ & $0.21 *$ & $0.25^{* *}$ & -0.13 \\
\hline & & $\begin{array}{c}\Delta R 8 \\
\mathrm{hPa} \cdot \mathrm{s} \cdot \mathrm{L}^{-1}\end{array}$ & $\begin{array}{c}\Delta X 8 \\
\mathrm{hPa} \cdot \mathrm{s} \cdot \mathrm{L}^{-1}\end{array}$ & $\underset{\mathrm{hPa} \cdot \mathrm{s} \cdot \mathrm{L}^{-1}}{\Delta \mathrm{FD}}$ & $\begin{array}{l}\Delta f 0 \\
\mathrm{~Hz}\end{array}$ \\
\hline \multicolumn{2}{|l|}{$\Delta \mathrm{FVC} 90-95 \quad \mathrm{~L}$} & -0.10 & 0.18 & 0.02 & -0.13 \\
\hline \multicolumn{2}{|l|}{$\triangle \mathrm{FEV}_{190-95} \mathrm{~L}$} & -0.08 & $0.20 *$ & 0.08 & $-0.22 * *$ \\
\hline \multicolumn{2}{|c|}{$\triangle \mathrm{FEV}_{1 / \mathrm{FVC} 90-95 \%} \%$} & 0.03 & 0.02 & 0.08 & -0.08 \\
\hline \multirow{2}{*}{\multicolumn{2}{|c|}{$\triangle \mathrm{PEF} 90-95 \quad \mathrm{~L} \cdot \mathrm{s}^{-1}$}} & 0.00 & -0.09 & 0.02 & 0.03 \\
\hline & & 0.00 & 0.70 & 0.06 & -0.07 \\
\hline
\end{tabular}

$\Delta$ : difference between lung function parameters (1990 minus 1995). Significances were tested by Pearson's correlation test. $*: \mathrm{p}<0.05$; **: $\mathrm{p}<0.01$. For further definitions see legend to table 1 .

of individual measurements with those at the start were much lower than parameters derived from flow-volume curves (table 1).

Airflow limitation (defined as an FEV1 <80\%) was present in $7(5.1 \%)$ of the subjects in 1990 and in 6 $(4.4 \%)$ of the subjects in 1995 . Three of these subjects had FEV1 $<80 \%$ predicted value in both the 1990 and 1995 groups. According to impedance criteria (a negative FD and $f 0$ exceeding $15 \mathrm{~Hz}$ ), airflow limitation was present in $5(3.8 \%)$ and $6(4.4 \%)$ of the subjects in 1990 and 1995, respectively. However, by this method only one subject was found to have airway obstruction at both time-points. No airway obstruction was detected by FOT among the seven workers identified as obstructive by flow-volume criteria in 1990, whilst it was present in only one of the six workers with airway obstruction measured by flow-volume in 1995 . To compare impedance and flow-volume measurements, correlation coefficients were again calculated (table 2). Although low, these coefficients showed quite similar values and patterns on both occasions. All impedance parameters were significantly correlated to FEV 1 and all but $f 0$ were correlated to MMEF. Only $R 8$ was correlated to FEV1/FVC. Changes in most impedance parameters were not related to longitudinal changes in flow-volume parameters. Only the change in reactance at $8 \mathrm{~Hz}$ was significantly $(p<0.05)$ correlated to longitudinal changes in FVC and FEV1, while $f_{0}$ increased significantly with the decline in FEV1 $(r=-0.22 ; \mathrm{p}<0.01)$.

Impedance parameters accompanying a (rapid) decline in FEVI

To investigate a relationship between longitudinal changes in FEV1 and impedance parameters, the total group 
was divided into four groups (quartiles) of decline in FEV1, with an approximately equal number of subjects in each (table 3). The data show that a decline in FEV1 is accompanied by similar changes in other flow-volume parameters (FVC, FEV1/FVC ratio, PEF, and MMEF). Similarly, impedance parameters, such as $f 0$ and $R 8$ tended to increase in relation to increasing decline in FEV1, whereas $X 8$ and FD tended to decrease. Trends were considered significant if those in group IV $(\triangle \mathrm{FEV} 1$ $>330 \mathrm{~mL}$ during the $5 \mathrm{yrs}$ ) showed a significant difference (ANOVA, $\mathrm{p}<0.05$ ) compared to the others. This was found for $f 0$ in 1995, the decline in reactance at 8 $\mathrm{Hz}(\Delta X 8)$, and the increase in resonance frequency $(\Delta f 0)$. After correction for age, all longitudinal changes in flow-volume parameters $(\triangle \mathrm{FVC}, \triangle \mathrm{FEV} 1 / \mathrm{FVC}, \triangle \mathrm{PEF}$ and $\triangle \mathrm{MMEF}$ ) in group I were still significantly different (logistic regression, all $\mathrm{p}<0.01)$. Flow-volume parameters $(\triangle \mathrm{FVC}, \triangle \mathrm{FEV} 1 / \mathrm{FVC}$, and $\triangle \mathrm{MMEF})$ and $\Delta R 8$ in group III were no longer significantly different. Interestingly, in a linear regression model, the decline in FEV1 $(\triangle \mathrm{FEV} 1)$ was significant $(\mathrm{r}=0.35 ; \mathrm{p}=0.01)$ by a model using age, years of employment, pack-years, FEV1, $R 8, X 8, \mathrm{FD}$ and $f 0$ at the starting point $(\triangle \mathrm{FEV} 1=-0.70+0.01$ age -0.01 yrs of employment +0.001 pack-years $+0.12 \mathrm{FEV} 1,1990$ + $0.02 R 8,1990-0.09 X 8,1990-0.02$ FD1990 - $0.01 f 0,1990)$. Similarly, alterations in $f 0$ were significantly dependent $(r=0.56 ; p<0.01)$ on age $(\beta=-0.04)$, years of employment $(\beta=0.10)$, pack-years $(\beta=0.03), f_{0}(\beta=0.61), F_{1}(\beta=0.11)$, $\operatorname{FVC}(\beta=0.56)$, PEF $(\beta=-0.02)$ and MMEF $(\beta=0.62)$ at the starting point. Although there was no significant difference in smoking habits and most of the respiratory symptoms among the four quartiles of FEV1 alteration, group IV had more complaints of shortness of breath $(\mathrm{p}<0.05)$.

\section{Exposure and lung function (decline)}

Since the number of individuals with airway obstruction (FEV1 $<80 \%$ pred) was small (6 out of 136 ), the risk of specific exposures was evaluated using the presence of subjects with rapid decline in FEV1 (upper quartile). Comparison of lung function parameters in 1995 between the different plants (table 4) showed significant airway obstruction $(\mathrm{p}<0.01)$ in the bulk/transport workers $(n=14)$ compared to the other groups both based on flow-volume (FEV1, FVC) and impedance parameters $(X 8, f 0)$, but $R 8$ and FD were not significantly changed (data not shown). At the start (1990), bulk/transport workers also had lower flow-volume parameters $(\mathrm{p}<0.05)$ but impedance parameters were still normal compared to other plants. A lower FEV1 in this group (3.40 versus $3.86 \mathrm{~L}$ in the rest) is again associated with a higher $f 0$ (10.08 versus $7.72 \mathrm{~Hz}$ ) and a lower $X 8$ (-0.05 versus 0.19 $\left.\mathrm{hPa} \cdot \mathrm{s} \cdot \mathrm{L}^{-1}\right)$. Phlegm was reported more frequently $(\mathrm{p}<0.04)$ in bulk/transport workers (4 out of 10) than in the rest of the workers (11 out of 111), but no difference in symptoms were found between other groups. No difference in age, smoking, or years of employment was noted in this specific group; only one of the bulk/transport workers was initially employed in another production process and later transferred to this job, as compared to 28 out

Table 3. - Personal characteristics, flow-volume and impedance parameters and respiratory complaints of subjects classified into quartiles of decline in FEV 1

\begin{tabular}{|c|c|c|c|c|c|}
\hline Parameters & Group I & Group II & Group III & Group IV & p-value ${ }^{+}$ \\
\hline $\mathrm{n}$ & 34 & 35 & 33 & 34 & \\
\hline Age yrs & $38^{*}$ & 39 & $43 * *$ & 43 & 0.03 \\
\hline Employment yrs & $14.4 \pm 7.8$ & $15.5 \pm 7.7$ & $18.7 \pm 7.6$ & $18.1 \pm 8.4$ & 0.08 \\
\hline Cigarettes $n \cdot$ day $^{-1}$ & $3.8 \pm 8.0$ & $2.2 \pm 5.8$ & $4.1 \pm 7.1$ & $4.0 \pm 7.0$ & 0.64 \\
\hline Pack-yrs & $7.5 \pm 9.0$ & $5.2 \pm 7.1$ & $9.3 \pm 11.4$ & $9.7 \pm 10.8$ & 0.20 \\
\hline Smokers $\mathrm{n}(\%)$ & $8(24)$ & $7(20)$ & $10(30)$ & $11(32)$ & 0.62 \\
\hline \multicolumn{6}{|l|}{ Flow-volume } \\
\hline$\Delta \mathrm{FVC} \mathrm{L}$ & $-0.10 \pm 0.24 * *$ & $0.07 \pm 0.27$ & $0.12 \pm 0.25^{*}$ & $0.29 \pm 0.29 * *$ & $<0.01$ \\
\hline$\Delta \mathrm{FEV}_{1} / \mathrm{FVC} \%$ & $0.53 \pm 3.75^{* *}$ & $1.53 \pm 3.89$ & $3.31 \pm 4.31 * *$ & $4.60 \pm 4.05^{* *}$ & $<0.01$ \\
\hline$\triangle \mathrm{PEF} \quad \mathrm{L} \cdot \mathrm{s}^{-1}$ & $-0.37 \pm 1.30 * *$ & $0.09 \pm 1.51$ & $0.25 \pm 0.99$ & $0.78 \pm 1.44 * *$ & $<0.01$ \\
\hline$\triangle \mathrm{MMEF} \quad \mathrm{L} \cdot \mathrm{s}^{-1}$ & $0.18 \pm 0.42 * *$ & $0.48 \pm 0.41$ & $0.68 \pm 0.55 * *$ & $0.89 \pm 0.58 * *$ & $<0.01$ \\
\hline \multicolumn{6}{|l|}{ Impedance } \\
\hline$R 8 \mathrm{hPa} \cdot \mathrm{S} \cdot \mathrm{L}^{-1}$ & $2.35 \pm 0.68$ & $2.35 \pm 0.84$ & $3.31 \pm 4.89$ & $2.67 \pm 0.89$ & 0.35 \\
\hline$X 8 \mathrm{hPa} \cdot \mathrm{s} \cdot \mathrm{L}^{-1}$ & $0.22 \pm 0.35$ & $0.15 \pm 0.28$ & $0.16 \pm 0.27$ & $0.11 \pm 0.29$ & 0.53 \\
\hline $\mathrm{FD} h \mathrm{hPa} \cdot \mathrm{s} \cdot \mathrm{L}^{-1}$ & $0.28 \pm 0.56$ & $0.39 \pm 0.65$ & $0.13 \pm 0.54$ & $0.25 \pm 0.74$ & 0.40 \\
\hline$f_{0} \mathrm{~Hz}$ & $7.46 \pm 2.61$ & $7.77 \pm 2.98$ & $7.77 \pm 2.21$ & $8.86 \pm 3.83 *$ & 0.23 \\
\hline$\Delta R 8 \quad \mathrm{hPa} \cdot \mathrm{s} \cdot \mathrm{L}^{-1}$ & $0.06 \pm 0.68$ & $-0.06 \pm 0.49$ & $-1.14 \pm 4.82 *$ & $-0.34 \pm 0.57$ & 0.18 \\
\hline$\Delta X 8 \quad \mathrm{hPa} \cdot \mathrm{s} \cdot \mathrm{L}^{-1}$ & $-0.06 \pm 0.37$ & $-0.06 \pm 0.22$ & $0.00 \pm 0.20$ & $0.07 \pm 0.23 *$ & 0.10 \\
\hline$\Delta \mathrm{FD} \quad \mathrm{hPa} \cdot \mathrm{s} \cdot \mathrm{L}^{-1}$ & $0.27 \pm 0.67$ & $0.13 \pm 0.58$ & $0.39 \pm 0.60$ & $0.26 \pm 0.75$ & 0.47 \\
\hline$\Delta f 0 \mathrm{~Hz}$ & $-0.01 \pm 3.19$ & $0.69 \pm 2.24 *$ & $-0.40 \pm 2.90$ & $-1.61 \pm 3.34 * *$ & 0.01 \\
\hline \multicolumn{6}{|l|}{ Complaints } \\
\hline Cough n (\%) & $4(12)$ & $2(6)$ & $6(18)$ & $2(6)$ & \\
\hline Phlegm n (\%) & $3(9)$ & $2(6)$ & $7(21)$ & 3 (9) & \\
\hline Wheezing $\mathrm{n}(\%)$ & $6(18)$ & $8(23)$ & $8(24)$ & $6(18)$ & \\
\hline Shortness of breath $\mathrm{n}(\%)$ & $5(15)$ & $3(9)$ & $10(30)$ & $11(32)^{*}$ & 0.03 \\
\hline
\end{tabular}

Values are presented as mean \pm SD. Group I $(\Delta \mathrm{FEV} 1<0.06 \mathrm{~L})$; Group II $(0.06<\Delta \mathrm{FEV} 1<0.20 \mathrm{~L})$; Group III $(0.20<\Delta \mathrm{FEV} 1<0.33$ L); Group IV ( $\Delta \mathrm{FEV} 1>0.33 \mathrm{~L})$. $\Delta$ : difference between lung function parameters (1990 minus 1995). Significances were tested by one-way analysis of variance (ANOVA) and tested between a specific group versus the other groups, if significant; *: $p<0.05$; **: p $<0.01$. Note: negative changes indicate increases from $1990-1995$, positive changes indicate decrease. ${ }^{+}$: for comparison across all groups. For further definitions see legend to table 1 . 
Table 4. - Characteristics and lung function outcomes of workers from different production processes

\begin{tabular}{|c|c|c|c|c|c|c|c|c|}
\hline \multicolumn{2}{|l|}{ Parameter } & $\begin{array}{c}\text { Chemicals } \\
(n=38)\end{array}$ & $\begin{array}{l}\text { Acids } \\
(\mathrm{n}=18)\end{array}$ & $\begin{array}{l}\text { Polymers } \\
(\mathrm{n}=12)\end{array}$ & $\begin{array}{l}\text { Polyurethanes } \\
\qquad(\mathrm{n}=21)\end{array}$ & $\begin{array}{l}\text { Bulk/Transport } \\
\qquad(\mathrm{n}=14)\end{array}$ & $\begin{array}{l}\text { Maintenance } \\
\quad(\mathrm{n}=10)\end{array}$ & $\begin{array}{l}\text { Others } \\
(n=23)\end{array}$ \\
\hline \multicolumn{2}{|c|}{$\begin{array}{l}\text { Age yrs } \\
\text { Employment yrs } \\
\text { Smoking +/- } \\
\text { Pack-years }\end{array}$} & $\begin{array}{c}41 \pm 8 \\
17.8 \pm 7.8 \\
30 / 8 \\
8.2 \pm 8.7\end{array}$ & $\begin{array}{c}41 \pm 9 \\
17.0 \pm 8.2 \\
12 / 6 \\
9.7 \pm 10\end{array}$ & $\begin{array}{c}36 \pm 10 \\
12.9 \pm 9.3 \\
8 / 4 \\
9.1 \pm 12.9\end{array}$ & $\begin{array}{c}38 \pm 7 \\
13.7 \pm 7.1 \\
10 / 11 \\
5.0 \pm 7.7\end{array}$ & $\begin{array}{c}41 \pm 7 \\
16.6 \pm 9.0 \\
9 / 5 \\
7.6 \pm 9.9\end{array}$ & $\begin{array}{c}45 \pm 7 \\
21.6 \pm 7.2 * \\
4 / 6 \\
5.0 \pm 10.1\end{array}$ & $\begin{array}{c}43 \pm 10 \\
17.0 \pm 7.2 \\
14 / 9 \\
9.4 \pm 11.1\end{array}$ \\
\hline $\begin{array}{l}\text { FVC L } \\
\triangle F V C \\
\text { FEV1 L } \\
\triangle F^{\prime} V_{1}\end{array}$ & $\begin{array}{l}1990 \\
1995 \\
\\
1990 \\
1995\end{array}$ & $\begin{array}{l}5.24 \pm 0.65 \\
5.10 \pm 0.66 \\
0.13 \pm 0.27 \\
4.01 \pm 0.51 \\
3.81 \pm 0.48 \\
0.19 \pm 0.16\end{array}$ & $\begin{array}{l}5.52 \pm 0.69 \\
5.36 \pm 0.77 \\
0.16 \pm 0.28 \\
4.20 \pm 0.58 \\
3.97 \pm 0.60 \\
0.23 \pm 0.20\end{array}$ & $\begin{array}{c}5.18 \pm 0.71 \\
5.27 \pm 0.69 \\
-0.08 \pm 0.35^{*} \\
4.10 \pm 0.52 \\
3.99 \pm 0.56 \\
0.10 \pm 0.23\end{array}$ & $\begin{array}{c}5.32 \pm 0.58 \\
5.36 \pm 0.59 \\
-0.03 \pm 0.32 * \\
4.17 \pm 0.52 \\
3.99 \pm 0.53 \\
0.17 \pm 0.14\end{array}$ & $\begin{array}{l}4.84 \pm 0.69 * * \\
4.71 \pm 0.72 * * \\
0.12 \pm 0.30 \\
3.68 \pm 0.67 * \\
3.40 \pm 0.68 * * \\
0.27 \pm 0.29\end{array}$ & $\begin{array}{l}5.18 \pm 0.60 \\
4.90 \pm 0.57 \\
0.27 \pm 0.16 \\
3.86 \pm 0.64 \\
3.60 \pm 0.67 \\
0.26 \pm 0.18\end{array}$ & $\begin{array}{l}5.33 \pm 0.87 \\
5.23 \pm 0.93 \\
0.10 \pm 0.29 \\
3.99 \pm 0.77 \\
3.80 \pm 0.80 \\
0.18 \pm 0.26\end{array}$ \\
\hline $\begin{array}{l}X 8 \\
\Delta X 8\end{array}$ & $\begin{array}{l}1990 \\
1995\end{array}$ & $\begin{array}{r}0.11 \pm 0.33 \\
0.19 \pm 0.38 \\
-0.07 \pm 0.40\end{array}$ & $\begin{array}{l}0.15 \pm 0.23 \\
0.08 \pm 0.33 \\
0.07 \pm 0.20\end{array}$ & $\begin{array}{r}0.17 \pm 0.20 \\
0.24 \pm 0.15 \\
-0.07 \pm 0.14\end{array}$ & $\begin{array}{r}0.18 \pm 0.16 \\
0.21 \pm 0.18 \\
-0.05 \pm 0.14\end{array}$ & $\begin{array}{c}0.06 \pm 0.16 \\
-0.05 \pm 0.23 * * \\
0.11 \pm 0.22 *\end{array}$ & $\begin{array}{l}0.19 \pm 0.23 \\
0.16 \pm 0.30 \\
0.02 \pm 0.15\end{array}$ & $\begin{array}{l}0.23 \pm 0.26 \\
0.23 \pm 0.25 \\
0.01 \pm 0.20\end{array}$ \\
\hline $\begin{array}{l}f_{0} \mathrm{~Hz} \\
\Delta f 0\end{array}$ & $\begin{array}{l}1990 \\
1995\end{array}$ & $\begin{array}{l}7.94 \pm 3.28 \\
7.88 \pm 3.00 \\
0.06 \pm 3.87\end{array}$ & $\begin{array}{r}8.61 \pm 4.19 \\
8.86 \pm 3.63 \\
-0.25 \pm 2.75\end{array}$ & $\begin{array}{l}7.29 \pm 2.01 \\
7.28 \pm 2.24 \\
0.00 \pm 0.99\end{array}$ & $\begin{array}{r}6.87 \pm 1.84 \\
7.07 \pm 1.87 \\
-0.20 \pm 2.36\end{array}$ & $\begin{array}{c}7.68 \pm 1.11 \\
10.08 \pm 3.35^{* *} \\
-2.39 \pm 2.94^{* *}\end{array}$ & $\begin{array}{r}7.30 \pm 1.29 \\
7.97 \pm 2.74 \\
-0.65 \pm 1.69\end{array}$ & $\begin{array}{l}7.38 \pm 2.91 \\
7.29 \pm 2.99 \\
0.09 \pm 3.17\end{array}$ \\
\hline $\begin{array}{l}\text { RR } \\
95 \% \text { CI }\end{array}$ & & $\begin{array}{c}0.73 \\
0.30-1.81\end{array}$ & $\begin{array}{c}2.14 \\
0.75-6.07\end{array}$ & $\begin{array}{c}0.25 \\
0.03-2.01\end{array}$ & $\begin{array}{c}0.45 \\
0.12-1.64\end{array}$ & $\begin{array}{c}1.78 \\
0.55-5.74\end{array}$ & $\begin{array}{c}2.13 \\
0.56-8.06\end{array}$ & $\begin{array}{c}1.07 \\
0.38-2.98\end{array}$ \\
\hline
\end{tabular}

Values are presented as mean \pm SD. RR: relative risk presence of rapid decliners in FEV1 (upper quartile) in the different plants; CI: 95\% confidence interval. Significances between a certain plant and the rest were tested by one-way analysis of variance (ANOVA). $*: \mathrm{p}<0.05 ; * *: \mathrm{p}<0.01 ; \Delta$ : differences between values in 1990 minus 1995 . For further definitions see legend to table 1 . Note: negative changes indicate increases from 1990-1995, positive changes indicate decrease.

of 122 in the rest. No difference in lung function, age or employment duration was observed within the other "risk" groups (acids, maintenance). Maintenance workers, however, had been employed significantly longer $(21.6 \mathrm{yrs} ; \mathrm{p}<0.05)$ than the rest of this population $(16.3$ $y r s)$; they were also slightly older (45 yrs; $p=0.1$ ) than the rest (40 yrs). However, except for an increased FVC $(\%)$, no lung function differences were observed in the latter group. The relative risks (RR) calculated accordingly are presented in table 4. Although the table shows increased RR values for three production processes namely acids (2.14), bulk/transport (1.78) and maintenance (2.13), none of these values was statistically significant.

Table 5. - Influence of smoking habits on lung function parameters (1995)

\begin{tabular}{|c|c|c|c|c|}
\hline Parameter & $\begin{array}{c}\text { Current } \\
\text { smokers } \\
(n=36)\end{array}$ & $\begin{array}{c}\text { Ex- } \\
\text { smokers } \\
(\mathrm{n}=51)\end{array}$ & $\begin{array}{c}\text { Nonsmokers } \\
\qquad(\mathrm{n}=49)\end{array}$ & $\mathrm{p}$-value \\
\hline \multicolumn{5}{|l|}{ Flow-volume } \\
\hline FVC L & $5.11 \pm 0.84$ & $5.01 \pm 0.64$ & $5.34 \pm 0.72^{\#}$ & 0.07 \\
\hline FEV1 L & $3.63 \pm 0.71 *$ & $3.37 \pm 0.49$ & $4.07 \pm 0.61^{\# \#}$ & ${ }^{*}<0.01$ \\
\hline FEV1/FVC \% & $71 \pm 8 * *$ & $74 \pm 5$ & $76 \pm 5^{\# \#}$ & $<0.01$ \\
\hline $\mathrm{PEF} \mathrm{L} \cdot \mathrm{s}^{-1}$ & $8.25 \pm 1.56^{*}$ & $8.67 \pm 1.58$ & $8.93 \pm 1.20$ & 0.10 \\
\hline MMEF L·s ${ }^{-1}$ & $2.74 \pm 1.00 * *$ & $3.05 \pm 0.83$ & $3.60 \pm 0.93^{\# \#}$ & ${ }^{*}<0.01$ \\
\hline$\Delta \mathrm{FEV}_{1} \mathrm{~L}$ & $0.24 \pm 0.22$ & $0.19 \pm 0.18$ & $0.17 \pm 0.22$ & 0.28 \\
\hline \multicolumn{5}{|l|}{ Impedance } \\
\hline$R 8 \mathrm{hPa} \cdot \mathrm{s} \cdot \mathrm{L}^{-1}$ & $3.25 \pm 4.71$ & $2.55 \pm 0.71$ & $2.36 \pm 0.79$ & 0.25 \\
\hline$X 8 \mathrm{hPa} \cdot \mathrm{s} \cdot \mathrm{L}^{-1}$ & $0.21 \pm 0.37$ & $0.13 \pm 0.28$ & $0.16 \pm 0.26$ & 0.47 \\
\hline $\mathrm{FD} \mathrm{hPa} \cdot \mathrm{s} \cdot \mathrm{L}^{-1}$ & $0.18 \pm 0.63$ & $0.16 \pm 0.63$ & $0.44 \pm 0.59^{\#}$ & 0.05 \\
\hline fo $\mathrm{Hz}$ & $7.57 \pm 2.88$ & $8.12 \pm 3.04$ & $8.09 \pm 3.04$ & 0.65 \\
\hline
\end{tabular}

Values are presented as mean \pm SD. For definitions see legends to tables 1 and 2. Significances were tested by one-way analysis of variance (ANOVA). Significant difference between current smokers vs ex- and nonsmokers, *: $\mathrm{p}<0.05 ; * *: \mathrm{p}<0.01$. Significant difference between nonsmokers $v s$ current and exsmokers, \#: $\mathrm{p}<0.05$; \#\#: $\mathrm{p}<0.01$.

\section{Smoking and lung function}

The effect of smoking on lung function was tested by one-way ANOVA among current smokers, ex-smokers and nonsmokers (table 5). Interestingly, the data show significant differences $(\mathrm{p}<0.01)$ in most flow-volume (FEV1, FEV1/FVC and MMEF) parameters and only one impedance parameter $(\mathrm{FD} ; \mathrm{p}<0.05)$ between these groups. Moreover, the current smokers showed significantly lower FEV1 $(p<0.05), F E V 1 / F V C$ ratio $(p<0.01)$, PEF $(p<0.05)$ and MMEF $(p<0.01)$ compared to ex- and nonsmokers. The current and ex-smokers also showed a significant decrease in FVC $(\mathrm{p}<0.05), \mathrm{FEV} 1, \mathrm{FEV} 1 /$ FVC ratio and MMEF (all $\mathrm{p}<0.01$ ) compared to nonsmokers. Furthermore, nonsmokers have a lower decline in FEV1 compared to ex- and current smokers, although the difference was not statistically significant $(p=0.28)$. Impedance parameters revealed a similar pattern with smoking habits, although only FD was significantly different between groups. Current and ex-smokers had significantly lower FD $(\mathrm{p}<0.05)$ than nonsmokers.

\section{Discussion}

To our knowledge, this is the first report presenting follow-up data on impedance measurements in an occupational setting. We showed that most flow-volume and some impedance parameters changed significantly over a 5-year interval, and that alterations in impedance parameters reflect longitudinal changes in flow-volume curves. These data can be regarded as a valuable extension to the results found in cross-sectional studies in occupational settings. Although this study did not attempt to validate the FOT versus flow-volume measurements, it showed that in a population with normal changes in flowvolume parameters, changes in impedance parameters, 
although not always significant, followed these alterations as predicted by the model interpretation according to WOUTERs [10]. The lack of longitudinal significance is probably explained by the larger short-term and longterm variability in FOT derived parameters. A previous study in a small group $(n=10)$ of grain workers showed similar outcome at 2 year follow-up [13]. Individual repeatability of $R 8$ in our hands was slightly better compared to data published by NEILD et al. [14] for impedance at $3 \mathrm{~Hz}(\mathrm{Z3Hz})(11.3 \%)$ or STANESCU et al. [15] for resistance at $3-9 \mathrm{~Hz}(R 3-9 \mathrm{~Hz})(12.0 \%)$ for short-term variability. Our coefficients of variation for $R 8(10.5 \%), X_{8}$ $(14.6 \%)$, and $f_{0}(14.3 \%)$ would allow minimal detectable changes much smaller than those observed over 5 yrs in the present cohort. This probably means that long-term variation is larger in impedance than flow-volume parameters. This is in agreement with lower sensitivity of FOT compared to flow-volume. Although a high variability is present in frequency dependence, it is a very sensitive parameter to detect airway obstruction. This is, however, not based on the exact value, but upon the difference between a negative (airway obstruction) and a positive (normal) value.

A rapid decline in FEV1 was reflected in a significant increase in $f 0$ and a significant decrease in $X 8$. Meanwhile, $R 8$ increased considerably and FD decreased slightly. CAUBBerghs et al. [16], studying lung function during a follow-up of members of the Belgian Air Force, found no significant alterations in impedance parameters, and found diffusion capacity of the lung for carbon monoxide ( $D \mathrm{~L}, \mathrm{CO}), \mathrm{FEV} 1 / \mathrm{FVC}$ ratio and weight to be predictors of a rapid decline in lung function. In our study, initial flow-volume parameters together with age, pack-years and years of employment are the most important predictive factors of alterations in impedance parameters. Otherwise, initial impedance values together with age, pack-years and years of employment were also predictors of longitudinal decline in FEV1. A possible explanation for this finding might be that more sensitive parameters, $R 8$ and $X 8$, were used, as opposed to the total resistance and total reactance used by CAUBERGHS et al. [16]. Since airflow limitation is reflected by an increase in resistance and a decrease in reactance predominantly at lower frequencies [6, 10], total resistance and total reactance might introduce a significant amount of noise.

Our follow-up study shows that airflow obstruction is a reversible process in this group of workers, and the results show that reversibility is more frequently observed with impedance measurements. This suggests that, although changes in the outcome of both techniques are correlated, in part, different factors are being measured. This is illustrated firstly by the correlation coefficients of lung function parameters between both time-points, which are much lower for impedance measurements (0.23-0.53) than for flow-volume parameters (0.61-0.94). Secondly, correlations both between flow-volume and impedance measurements are consistent (table 2) but low. A similar finding was reported in workers from a gas manufacturing plant [7]. Interestingly, these correlation coefficients are much lower than those reported in asthmatics, where correlation coefficients up to 0.8 are usually observed [5]. Thirdly, changes in impedance parameters among subgroups classified with respect to decline in FEV1 are in line with model considerations
[10], but statistical significance remains limited to resonance frequency (tables 3 and 4). Finally, in agreement with other studies $[17,18]$, only frequency dependence is different between (ex) smokers and nonsmokers, while almost all flow-volume parameters differ between these groups.

The relative risk to airway obstruction due to specific exposures was evaluated using data on lung function at follow-up and its decline during follow-up, since the number of obstructive subjects $(\mathrm{FEV} 1<80 \%)$ was too small. The analysis showed a relative excess of rapid decliners among maintenance workers, bulk/transport workers and workers in the acid-producing plants. Confidence intervals, however, were wide due to the small size of the cohort and, therefore, relative risks are not statistically significant. Interestingly, only in bulk/transport workers were average lung function data (both impedance and flow-volume) significantly different from the other groups. This result is unlikely to be the effect of selection and transfer of unhealthy workers from other plants, as shown by the internal transfer records (data not shown). Confounding by smoking is also unlikely. Firstly, no differences in smoking habits were noted between different groups. Secondly, the impedance parameter mostly affected by smoking, i.e. frequency dependence (table 5), is not changed in bulk transport workers. Conversely, $X 8$ and $f 0$, which are not affected by smoking, were significantly different in this group. The present findings in bulk/transport workers are probably explained by their high exposure to "inert" NPK-dust (5-25 $\left.\mathrm{mg} \cdot \mathrm{m}^{-3}\right)$ present in the fertilizer production unit. These outcomes are in good agreement with a previous cross-sectional study in potato sorters exposed to high concentrations of inert dust $\left(6.9-13.3 \mathrm{mg} \cdot \mathrm{m}^{-3}\right)$, being diatomaceous earth [19]. In that study also, higher $R 8$ and $f 0$ values were observed in exposed workers compared to controls, and a lower FEV1, MMEF and X8.

Findings in maintenance and acid-producing workers are probably explained by their typical exposure pattern, consisting of single peak exposures to irritants and vapours during (un)loading, fitting and repair. It has been reported that intermittent peak exposures to airway irritants can cause airways hyperresponsiveness (AHR) [20], and that the number of peak exposures can be a better descriptor of lung function [21] than the average exposure. A recent study on AHR in 688 male workers exposed to low-level irritants [22] showed a lower prevalence of AHR in subjects with less than 5 yrs exposure to $\mathrm{SO}_{2}$ and oil mist, probably due to pre-employment screening. No association between AHR and low-grade exposure to irritants, however, could be established. BROCHARD et al. [7] observed normal flow-volume curves but altered impedance values in a group of 36 workers exposed to respiratory irritants ( $>6$ yrs employment) in a gas manufacturing plant compared to the outcome of 46 nonexposed references ( $<6$ yrs employment) from the same plant. In the present cohort, not enough subjects with less than 5 yrs employment were present; however, a similar analysis using 7 yrs as a cut-off revealed a significant difference $(\mathrm{p}<0.05)$ in $X 8, \mathrm{FD}$ and $f 0$ in nonsmoking workers $(n=15)$ with less than 7 yrs employment compared to a similar group $(n=34)$ employed for more than 7 yrs. No differences in flow-volume parameters were present between these groups (data not shown). 
We conclude that although the forced oscillation technique is less sensitive compared to forced expiratory flow-volume measurements, it is a useful method for screening airway obstruction in this type of occupational exposure. Longitudinal changes in impedance parameters and effects of smoking and occupational exposure reflect or parallel changes in spirometric indices. A widespread application in occupational medicine will teach us the additional value of the forced oscillation technique in specific exposures.

Acknowledgements: The authors are seriously indebted to all workers for their participation in this study and to G. Sambaer for his assistance in flow-volume measurements and data retrieval. The authors also thank T.H.J.M. Jorna, R.P.F. Schins and T. Pijls for their assistance in the impedance measurement and data entry. SK is a fellow supported by the Indonesian SUDR project.

\section{References}

1. Làndsér FJ, Nagels J, Demedts $\mathrm{M}$, Billiet L, Van de Woestijne KP. A new method to determine frequency characteristics of the respiratory system. J Appl Physiol 1976; 41: 101-106.

2. Jorna THJM, Borm PJA, Wesseling GJ, Wouters EFM. Application of the forced oscillation technique (FOT) in different occupational settings. Eur Respir Rev 1994; $4: 19,155-158$.

3. Jorna THJM, Borm PJA, Maestrelli P, Fabbri LM, Wouters EFM. Localization of airway obstruction by impedance measurement in isocyanate-induced asthmatic reactions. Am Rev Respir Dis 1993; 147: A115.

4. Pairon JC, Iwatsubo Y, Hubert C, et al. Measurement of bronchial responsiveness by forced oscillation technique in occupational epidemiology. Eur Respir J 1994; 7: 484-489.

5. Wouters EFM, Polko AH, Visser BF. Response localization of the pharmacological agents histamine and salbutamol along the respiratory system by forced oscillations in asthmatic subjects. J Asthma 1989; 26: 185-193.

6. Ying Y, Peslin R, Duvivier C, Gallina C, Da Silva JF. Respiratory input and transfer mechanical impedances in patients with chronic obstructive pulmonary disease. Eur Respir J 1990; 3: 1186-1192.

7. Brochard L, Pelle G, Palmas J de, et al. Density and frequency dependence of resistance in early airway obstruction. Am Rev Respir Dis 1987; 135: 579-584.

8. Pham QT, Bourkard E, Chau N, et al. Forced oscillation technique (FOT): a new tool for epidemiology of occupational lung diseases? Eur Respir J 1995; 8: 1307-1313.
9. Jorna T, Borm PJA, Valks J, Houba R, Wouters EFM. Respiratory symptoms and lung function in animal feed workers. Chest 1994; 106: 1050-1055.

10. Wouters EFM. Total respiratory impedance measurement by forced oscillations: a noninvasive method to assess bronchial response in occupational medicine. Exp Lung Res 1990; 16: 25-40.

11. Orehek J, Nicoli MM, Delpierre S, Beaupré A. Influence of the previous deep inspiration on the spirometric measurement of provoked bronchoconstriction in asthma. Am Rev Respir Dis 1981; 123: 269-272.

12. Quanjer $\mathrm{PhH}$. Standardized lung function testing. Bull Eur Physiopathol Respir 1983; 19 (Suppl. 5): 1-95.

13. Borm PJA, Schins RPF, Derhaag TJJM, Kant IJ, Crossshift changes in blood inflammatory markers occur in the absence of airway obstruction in workers exposed to grain dust. Chest 1996; 109: 1078-1085.

14. Neild JE, Twort CHC, Chinn S, et al. The repeatibility and validity of respiratory resistance measured by the forced oscillation technique. Respir Med 1989; 83: 111118.

15. Stanescu DC, Fesler R, Variter C, Fans A, Brasseur L. A modified measurement of respiratory resistance by forced oscillation during normal breathing. J Appl Physiol 1975; 39: 305-311.

16. Cauberghs M, Clement J, van de Woestijne KP. Functional alterations accompanying a rapid decline in ventilatory function. Am Rev Respir Dis 1993; 147: 379-384.

17. Kjeldgaard JM, Hyde RW, Speers DM, Reichert WW. Frequency dependence of total respiratory resistance in early airway disease. Am Rev Respir Dis 1976; 114: 501-508

18. Hayes DA, Pimmel RL, Fulton JM, Bromberg PA. Cold air as bronchial provocation technique. Chest 1984; 86 : 810-814.

19. Jorna THJM, Borm PJA, Koiter KD, Slangen JJM, Henderson P, Wouters EFM. Respiratory effects and serum type III procollagen in potato sorters exposed to diatomaceous earth. Int Arch Occup Environ Health 1994; 66: 217-222.

20. Brooks SM, Kalica AR. Strategies for elucidating the relationship between occupational exposures and chronic airflow obstruction. Am Rev Respir Dis 1987; 13: 268-273.

21. Tornling G, Alexandersson R, Hendenstierna G, Plato N. Decreased lung function and exposure to diisocyanates (HDI and HDI-BT) in car repair painters: observations on re-examination 6 years after initial study. Am J Ind Med 1990; 17: 299-310.

22. Kremer AM, Pal TM, Schouten JP, Rijcken B. Airway hyperresponsiveness in workers exposed to low levels of irritants. Eur Respir J 1995; 8: 53-61. 